(C) 2010 IEEE. Personal use of this material is permitted. Permission from IEEE must be obtained for all other uses, in any current or future media, including reprinting/republishing this material for advertising or promotional purposes, creating new collective works, for resale or redistribution to servers or lists, or reuse of any copyrighted component of this work in other works. 


\title{
Prioritized Independent Contact Regions for Form Closure Grasps
}

\author{
Robert Krug, Dimitar Dimitrov, Krzysztof Charusta and Boyko Iliev \\ AASS Research Center, Örebro University \\ Email: \{robert.krug, dimitar.dimitrov, krzysztof.charusta, boyko.iliev\}@oru.se
}

\begin{abstract}
The concept of independent contact regions on a target object's surface, in order to compensate for shortcomings in the positioning accuracy of robotic grasping devices, is well known. However, the numbers and distributions of contact points forming such regions is not unique and depends on the underlying computational method. In this work we present a computation scheme allowing to prioritize contact points for inclusion in the independent regions. This enables a user to affect their shape in order to meet the demands of the targeted application. The introduced method utilizes frictionless contact constraints and is able to efficiently approximate the space of disturbances resistible by all grasps comprising contacts within the independent regions.
\end{abstract}

\section{INTRODUCTION}

Accounting for uncertainties occurring in the positioning of robotic grasping devices is a central topic in dexterous manipulation research. The quality of a given multifingered grasp is not only reflected in its capability to withstand external disturbances, but also in its robustness to shortcomings in the positioning accuracy. An important issue in manufacturing and grasping procedures is object immobilization. In this context, Reuleaux [1] coined the term form-closure as the ability of a fixturing device to fully prevent motions of the target object via unilateral frictionless contact constraints. In contrast, force-closure imparts that the object's motion is restrained by suitable contact forces considering frictional contact constraints [2]. Contact force vectors and resulting torque vectors are commonly concatenated to wrench vectors. Mishra et al. [3] showed that a grasp is force/formclosure, if the convex hull spanned by the contact wrenches contains a neighborhood of the origin.

However, for many applications the basic ability to immobilize an object is just a necessary but not a sufficient requirement. A good grasp should be able to efficiently withstand disturbance forces/torques which are likely to occur during the performed task. If nothing about possible disturbances is known, a common grasp quality measure is the radius of the largest origin-centered insphere of the Grasp Wrench Space (GWS), which was proposed by Kirkpatrick et al. [4]. The GWS is defined as the set of all wrenches that a manipulator can exert on the object for a given grasp. In this definition it is presumed that the sum of the magnitude of the grasping forces is bounded.

From the viewpoint of a grasping device, not only the ability to resist disturbances, but also the robustness of a grasp to modeling and positioning inaccuracies is an important factor. In this context, the notion of Independent Contact Regions (ICRs) was introduced by Nguyen [5]. He defined the set of optimal independent regions with the largest minimal radius, which yield a force-closure grasp if each finger is placed anywhere within its respective region. The concept was extended to the computation of independent regions for three-finger grasps on planar objects [6] and four-finger grasps of polyhedral objects by Ponce et al. [7]. Pollard [8] employs the ICR paradigm in order to synthesize sets of similar whole-hand grasps on 3-D objects. The algorithms in [8] are based on geometric reasoning in the wrench space and incorporate a task related grasp quality measure. Roa and Suárez [9] suggested an approach which grows independent regions for precision grasps on discretized objects. In a previous work [10], the authors presented an efficient algorithm for the computation of such regions.

The approaches in [8][9][10] have in common that they utilize a prototype form/force-closure grasp and specify disturbances which need to be resisted. Eligible contact constraints can be formulated via frictionless/frictional or soft finger point contact models. ICRs are not unique with respect to the numbers and distributions of contact points forming the regions. Frequently, contact regions yielded by current methods are shaped undesirably. This is due to the fact that the underlying computational principle strongly conditions the shape of the resulting contact regions on the GWS of the given prototype grasp.

In this paper, we address the question of how to produce regions which are shaped to befit the considered application. For example regions comprising evenly distributed contact points are often attractive, since such uniform regions can be directly related to the positioning accuracy achievable by the deployed grasping device. Contributed is the idea of prioritizing contact points for inclusion in the ICRs. We limit ourselves to the form-closure case and utilize the frictionless point contact model. A computational method, which allows for a user to specify a suitable strategy for including contact points in the ICRs in order to form desired regions, is suggested. This novel approach allows to evaluate the actual disturbances every member of the grasp set associated with the ICRs is able to resist. To this end, we introduce the Exertable Wrench Space (EWS) as a generalization of the GWS applied to sets of grasps.

The assumptions and required background are provided in Section II. In sections III and IV we motivate and detail our computation scheme. Section V discusses possible prioritization strategies and finally in Section VI we provide a numerical evaluation of the suggested approach. 


\section{BACKGROUND}

\section{A. Nomenclature}

$\mathcal{S}$ Set of position vectors representing the target object,

$s$ index used for the points representing the object,

$N$ number of contact points in a grasp / number of ICRs,

$g_{n}$ index used for the $N$ grasp contact points,

$\mathcal{C}_{n} \quad$ set of contact points forming the $n^{\text {th }}$ ICR,

$\hat{\mathcal{C}}_{n} \quad$ set containing the indexes $c$ of the points in region $\mathcal{C}_{n}$,

$\mathcal{W}_{n}$ set of wrenches associated with the contact points in $\mathcal{C}_{n}$,

$H$ number of hyperplanes confining a convex hull,

$h$ index used for hyperplanes,

$K$ dimension of the wrench space.

\section{B. Assumptions \& Problem Description}

The target object's surface is described as a polygonal mesh (or a polygonal chain in the case of planar objects) whose vertices are represented by the set $\mathcal{S}=\left\{\boldsymbol{p}_{1}, \ldots \boldsymbol{p}_{S}\right\}$. The position vectors $\boldsymbol{p}_{s} \in \mathcal{S}$ are expressed in a coordinate frame which is located in the center of mass of the object. Each point $\boldsymbol{p}_{s}$ has an associated inward-pointing unit normal $\hat{\boldsymbol{n}}_{s}$ and neighboring points, defined as the ones connected to $\boldsymbol{p}_{s}$ by an edge of the mesh. Thus, this representation can be seen as a graph where nodes represent mesh vertices $\boldsymbol{p}_{s}$ and edges define the neighboring relation between these vertices. A grasp $\mathcal{G}=\left\{\boldsymbol{p}_{g_{1}}, \ldots, \boldsymbol{p}_{g_{N}}: \boldsymbol{p}_{g_{n}} \in \mathcal{S}\right\}$ is represented as a set of $N$ contact points. ICRs are specified as $N$ sets $\mathcal{C}_{n}$ which contain points on the target object's surface. Each finger of a grasping device is associated with one such region. Form-closure grasps, which comprise one contact drawn from each region $\mathcal{C}_{n}$ and are suitable to resist expected disturbances (see Section II-D), are denoted as viable grasps. It is assumed that the target object is sufficiently discretized to capture local curvature, i.e., grasps with contacts on mesh facets spanned by the discrete points forming regions $\mathcal{C}_{n}$ are also viable grasps. We presume that user-input in form of an initial viable grasp $\mathcal{G}_{\text {init }}$ is available. The necessary prototype grasp can be acquired by one of the many algorithms proposed for grasp synthesis (e.g. [9][11]) or by an expert demonstrator. Furthermore, quasi-static conditions are assumed and the kinematic constraints of the device grasping the target object are not considered.

The aim of this work is to allow a user to influence the distribution of contact points within the regions $\mathcal{C}_{n}$, i.e., to affect their shape on the target object's boundary. In addition to the necessary initial grasp $\mathcal{G}_{\text {init }}$ and expected disturbances, we consider two possible user inputs. First, we want to investigate what is the set of disturbances that every viable grasp is guaranteed to resist, if desired regions $\mathcal{C}_{n}$ themselves are defined by a user beforehand. An example of predefined ICRs for an eight-fingered grasp on the model of a parallelepiped is shown in Fig. 1. Second, we are interested in prioritizing contact points for inclusion in regions $\mathcal{C}_{n}$ according to a user-provided logic.

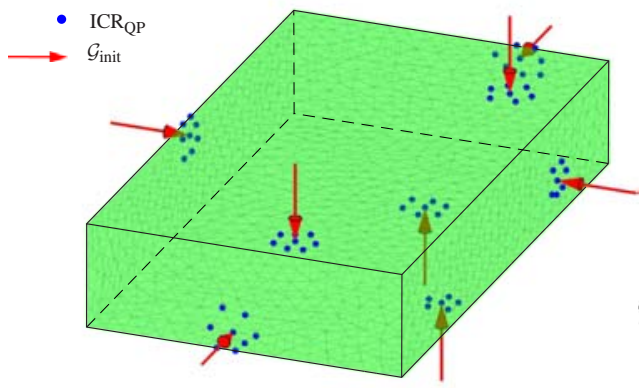

Fig. 1. Predefined Contact Regions on a parallelepiped: Uniform regions $\mathcal{C}_{n}$ for an 8-fingered grasp; Contact points in the Regions $\mathcal{C}_{n}$ are specified via a BFS, stopping at a depth of one (see Section V-A); Form-closure of all viable grasps was verified utilizing Algorithm 1;

\section{Contact Model}

We consider frictionless point contacts between the target object and the fingers of the gripper. The force $f_{s}$ applied at point $\boldsymbol{p}_{s}$ acts along the normal $\hat{\boldsymbol{n}}_{s}$ and creates a torque $\boldsymbol{\tau}_{s}=\boldsymbol{p}_{s} \times \boldsymbol{f}_{s}$. Force and torque vectors can be concatenated to a wrench vector $\boldsymbol{w}_{s}=\left(\boldsymbol{f}_{s}, \boldsymbol{\tau}_{s} / \lambda\right)$. Dividing the torque parts by the largest possible torque arm $\lambda=\max _{s}\left(\left\|\boldsymbol{p}_{s}\right\|\right)$ grants scale invariance [12]. Let $\hat{\mathcal{C}}_{n}$ be the set containing indexes of the contact points forming the $n^{\text {th }}$ independent region. Thereby, the region formed by these contact points can be formalized as $\mathcal{C}_{n}=\left\{\boldsymbol{p}_{c}: c \in \hat{\mathcal{C}}_{n}\right\}$. The corresponding wrench set is defined as

$$
\mathcal{W}_{n}=\left\{\boldsymbol{w}\left(\boldsymbol{p}_{c}\right): c \in \hat{\mathcal{C}}_{n}\right\} .
$$

Given a grasp $\mathcal{G}$, the discrete grasp wrench space in the frictionless case is described by the convex hull over the $N$ wrenches generated at the grasping points $\boldsymbol{p}_{g_{n}}$.

$$
\text { GWS }=\text { ConvHull }\left(\left\{\boldsymbol{w}\left(\boldsymbol{p}_{g_{1}}\right), \ldots, \boldsymbol{w}\left(\boldsymbol{p}_{g_{N}}\right)\right\}\right) .
$$

Note that we assume convex hulls to be in simplicial form. Equation (2) characterizes the space of wrenches, which can be exerted to the grasped object when the sum of the magnitudes of all finger forces is bounded. All wrenches lie on the Limit Wrench Space (LWS) [12], which is limited only by the magnitude of the contact forces (unit magnitude in the present case) and the resulting torques.

\section{State of the art}

Recent approaches for generating ICRs [8][9][10] allow for user-input in form of a set comprising expected disturbance wrenches. The convex hull over the mirror image of this set is commonly labeled as Task Wrench Space (TWS). It represents the set of wrenches which every viable grasp has to be able to exert on the object in order to counterbalance the expected disturbances and is frequently represented as an origin-centered sphere. Figure 2 illustrates the basic principle of the current methods. Shown is the computation procedure in a hypothetical 2-D wrench space for a fourfingered frictionless grasp. The ICRs obtained depend on the geometric properties of the grasp wrench space $\mathrm{GWS}_{\text {init }}$, corresponding to a provided initial prototype force-closure grasp $\mathcal{G}_{\text {init }}$, and the disturbances considered via the TWS. By construction, the TWS is a subset of the space of wrenches 


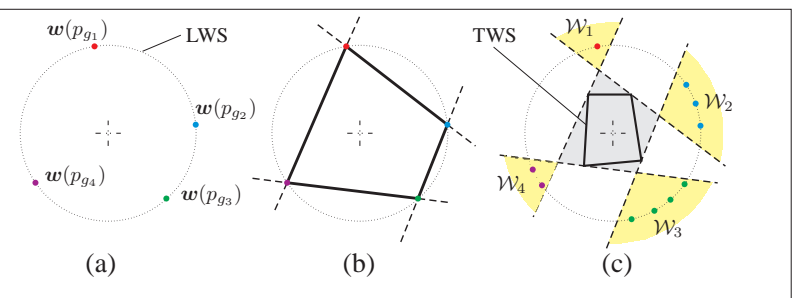

Fig. 2. Forming search regions via parallel shifting: (a) Wrenches associated with the initial grasp $\mathcal{G}_{\text {init }}$; (b) The corresponding $\mathrm{GWS}_{\text {init }}$ and its confining hyperplanes; (c) The TWS represents the set of wrenches necessary to counterbalance expected disturbances; Hyperplanes associated

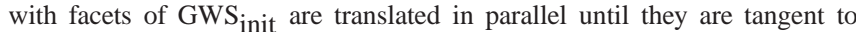
the TWS; Search spaces (the yellow shaded areas) in the wrench space are formed by the intersection of exterior half-spaces ${ }^{1}$ corresponding to the shifted hyperplanes; A point $\boldsymbol{p}_{s}$ on the object's boundary qualifies for inclusion in region $\mathcal{C}_{n}$ if its associated wrench lies in the search space associated with $\boldsymbol{p}_{g_{n}}$; Regions $\mathcal{C}_{n}$ on the object's surface are formed by the contact points with associated wrenches in the sets $\mathcal{W}_{n}$;

which is guaranteed to be exertable by every viable grasp (the gray shaded area in Fig. 2-c). A consequence of this method is that the resulting contact regions $\mathcal{C}_{n}$ are strongly conditioned on the structure of $\mathrm{GWS}_{\text {init }}$. In a significant number of cases, the number and distribution of contact points forming these regions might not be desirable for the targeted application.

\section{The Exertable Wrench Space (EWS)}

Let us for the moment assume that the ICRs are given beforehand and let $V$ denote the number of associated viable grasps. If the sets $\mathcal{C}_{n}$ forming the ICRs are disjoint, $V$ is given by the product of the cardinalities of $\mathcal{C}_{n}$.

$$
V=\prod\left|\mathcal{C}_{n}\right|, \quad n=1, \ldots, N .
$$

In the case of partially intersecting sets $\mathcal{C}_{n}$, which is admissible in the scope of this paper, the number $V$ gives an upper bound. This allows for a formal definition of the exertable wrench space as the intersection of all grasp wrench spaces $\mathrm{GWS}_{v}$ corresponding to viable grasps.

$$
\mathrm{EWS}=\bigcap\left\{\mathrm{GWS}_{1}, \ldots, \mathrm{GWS}_{V}\right\} .
$$

Analogue to the GWS, which is composed of the wrenches a single grasp can exert, the EWS represents the space of wrenches which every grasp in the set of viable grasps at least can apply to the target object. Figure 3 shows the relation between the EWS and the contact points in the ICRs. If we consider to add additional contact points to regions $\mathcal{C}_{n}$, and thus to add additional wrenches to their associated sets $\mathcal{W}_{n}$ in (1), possibilities for new grasps emerge and the EWS is gradually limited since it is described by the intersection of all the GWS corresponding to these grasps.

The mirror image of the EWS provides an exact formulation of the disturbance wrenches each of the viable grasps $\mathcal{G}_{v}$ is guaranteed to withstand. However, from (3) and (4) it follows that a brute-force approach via computing and intersecting the convex hulls $\mathrm{GWS}_{v}$ of all possible grasps is prohibitive in the general case, since it requires the computation and intersection of $V$ convex hulls.

\footnotetext{
${ }^{1} \mathrm{~A}$ half-space is designated as exterior if it does not contain the origin, opposed to an interior half-space which contains the origin.
}

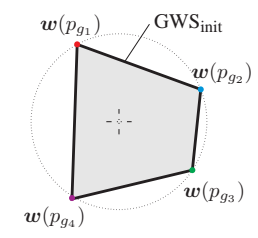

(a)

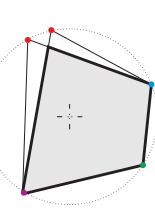

(b)

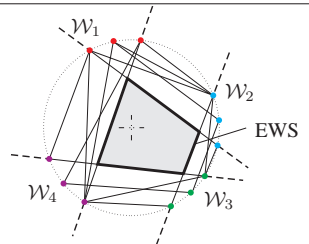

(c)
Fig. 3. Exertable wrench space: (a) The EWS equals the $\mathrm{GWS}_{\text {init }}$ if each region $\mathcal{C}_{n}$ only contains the initial grasping points $\boldsymbol{p}_{g_{n}}$; (b) Adding new points allows for new grasps to be formed by combinations of contact points in the sets $\mathcal{C}_{n}$; (c) The EWS is limited by the intersection of all grasp wrench spaces $\mathrm{GWS}_{v}$ corresponding to viable grasps (in this example not all of them are depicted for the sake of clarity); The facets of the EWS lie on hyperplanes which are spanned by wrenches from $K=2$ different sets $\mathcal{W}_{n}$ each; Those hyperplanes are coplanar to facets of the limiting $\mathrm{GWS}_{v}$;

\section{Evaluation of the EWS}

In this light, the general idea of the approaches discussed in Section II-D is to approximate the EWS based on parallel shifting of hyperplanes containing the facets of $\mathrm{GWS}_{\text {init }}$. Points on the object's surface are qualified for inclusion in regions $\mathcal{C}_{n}$ if their associated wrenches lie in appropriate search spaces constructed in the wrench space. According to Pollard [8], this yields families of similar viable grasps. Here, we state an explicit definition of similarity opposed to the informal one given in [8]:

Definition 1: Two grasps are similar if the face lattices of their corresponding grasp wrench spaces are isomorphic.

Loosely speaking, the face lattice of a convex polytope describes its topology. The face lattice is the partially ordered set of all its faces, the ordering is by set inclusion. Thus, the above definition implies that the GWS of similar grasps comprise the same topological structure in a sense that there exists a homeomorphism between their faces. A more detailed discussion can be found in [13].

The state-of-the-art principle of approximating the confining hyperplanes of the EWS and forming associated search spaces provides a computationally efficient way of generating ICRs. However, it is by no means unique. The only requirement is that the intersection of interior halfspaces associated with the hyperplanes forming search spaces contains the TWS. This requirement is fulfilled by infinitely many hyperplanes if the constraint of parallelism is relaxed and inclination of these hyperplanes is allowed. In the following, we motivate a strategy for a better approximation of the EWS taking into account the wrench sets $\mathcal{W}_{n}$ corresponding to contact points in the specified desired independent regions $\mathcal{C}_{n}$. Let us formulate

Proposition 1: The facets of the EWS lie on hyperplanes, each spanned by wrenches from $K$ different sets $\mathcal{W}_{n}$.

According to (4), the EWS is formed by the intersection of all $\mathrm{GWS}_{v}$. Therefore, the facets of the EWS lie on hyperplanes which also contain facets of certain limiting $\mathrm{GWS}_{v}$ (see Fig. 3-c). Recall, that the facets of convex hulls are in simplicial form. Thus, the hyperplanes containing these facets have to be spanned by wrenches stemming from $K$ different sets by definition, which validates the above proposition. 


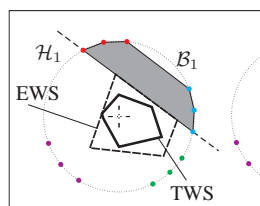

(a)

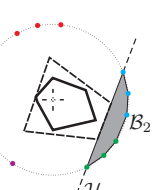

(b) ${ }^{\prime}$

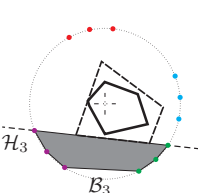

(c)

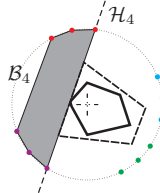

(d)
Fig. 4. Confining Hyperplanes of the EWS: Hyperplanes corresponding to facets of the convex hulls $\mathcal{B}_{h}$ confine the EWS; Those hyperplanes linearly separate the TWS and $\mathcal{B}_{h}$;

It is implied that the convex hull describing the $\mathrm{GWS}_{\text {init }}$ according to (2) is represented by its vertex-facet incidences which are stored in index sets $\xi_{h}, h=1, \ldots, H$. Thus, $\xi_{h}$ describes which vertices of GWS init $_{\text {span its }} h^{\text {th }}$ hyperplane. Consider the initial grasp wrench space depicted in Fig. 3-a. For this example the sets $\xi_{h}$ are: $\xi_{1}=\left\{\begin{array}{ll}1 & 2\end{array}\right\}, \xi_{2}=\left\{\begin{array}{ll}2 & 3\end{array}\right\}$, $\xi_{3}=\left\{\begin{array}{ll}3 & 4\end{array}\right\}$ and $\xi_{4}=\left\{\begin{array}{ll}1 & 4\end{array}\right\}$. We define the $H$ unions of wrench sets $\mathcal{W}_{n}$ in (4) indexed by $\xi_{h}$ as

$$
\mathcal{W}_{\xi_{h}}=\bigcup \mathcal{W}_{n}: n \in \xi_{h}, \quad h=1, \ldots, H
$$

Let $\mathcal{B}_{h}=$ ConvHull $\left(\mathcal{W}_{\xi_{h}}\right), h=1, \ldots, H$ describe the convex hulls over the wrench sets formulated above. The facets of the EWS lie on hyperplanes which also contain facets of the convex hulls $\mathcal{B}_{h}$ as depicted in Fig. 4. In the following, the polytope $\mathrm{EWS}_{\mathrm{app}}$, formed by the intersection of interior half-spaces defined by hyperplanes $\widetilde{\mathcal{H}}_{h}$, denotes an approximation of the actual EWS. Eligible hyperplanes $\widetilde{\mathcal{H}}_{h}$ need to linearly separate $\mathcal{B}_{h}$ from the TWS (which has to be a subset of EWS $\mathrm{Epp}_{\text {ap }}$. In order for hyperplanes $\widetilde{\mathcal{H}}_{h}$ to approximate the hyperplanes confining the EWS well, we want to find those $\widetilde{\mathcal{H}}_{h}$ which maximize the margins between the TWS and the convex hulls $\mathcal{B}_{h}$.

\section{A. Approximation of the EWS}

An efficient solution of the problem of finding the maximum-margin separating hyperplane between two convex hulls is provided in the framework of Support Vector Machines (SVM) [14]. Let $\boldsymbol{W}_{\xi_{h}} \in \mathbb{R}^{M \times K}$ be the matrix corresponding to $\mathcal{W}_{\xi_{h}}$ in (5), where $M=\sum\left|\mathcal{C}_{n}\right|: n \in \xi_{h}$. $\boldsymbol{W}_{\text {TWS }} \in \mathbb{R}^{T \times K}$ denotes the matrix whose rows contain the $T$ wrenches defining the TWS. Stated below is the convex Quadratic Program (QP) used to solve the separation problem

$$
\begin{aligned}
\underset{\boldsymbol{n}_{h}^{\prime} \in \mathbb{R}^{K}, b_{h}^{\prime} \in \mathbb{R}}{\operatorname{minimize}} & \frac{1}{2}\left[\begin{array}{c}
\boldsymbol{n}_{h}^{\prime} \\
b_{h}^{\prime}
\end{array}\right]^{T}\left[\begin{array}{cc}
\boldsymbol{I} & \mathbf{0} \\
\mathbf{0} & 0
\end{array}\right]\left[\begin{array}{c}
\boldsymbol{n}_{h}^{\prime} \\
b_{h}^{\prime}
\end{array}\right] \\
\text { subject to } & {\left[\begin{array}{cr}
-\boldsymbol{W}_{\xi_{h}} & -\mathbb{1} \\
\boldsymbol{W}_{\mathrm{TWS}} & \mathbb{1}
\end{array}\right]\left[\begin{array}{c}
\boldsymbol{n}_{h}^{\prime} \\
b_{h}^{\prime}
\end{array}\right] \geq\left[\begin{array}{c}
\mathbb{1} \\
\mathbb{1}
\end{array}\right], }
\end{aligned}
$$

where $\mathbb{1}$ denotes column vectors of ones with appropriate dimensions. The hyperplane $\widetilde{\mathcal{H}}_{h}$ tangent to $\mathcal{B}_{h}$ is defined by the unit vector $\boldsymbol{n}_{h}$ and the offset $b_{h}$ given below.

$$
\boldsymbol{n}_{h}=\frac{\boldsymbol{n}_{h}^{\prime}}{\left\|\boldsymbol{n}_{h}^{\prime}\right\|}, \quad b_{h}=\frac{1+b_{h}^{\prime}}{\left\|\boldsymbol{n}_{h}^{\prime}\right\|} .
$$

The only slight deviation from the conventional SVM formulation lies in the offset value $b_{h}$ in (7) which causes $\widetilde{\mathcal{H}}_{h}$ being tangent to $\mathcal{B}_{h}$, whereas the offset in the standard formulation would be $b_{h}^{\prime} /\left\|\boldsymbol{n}_{h}^{\prime}\right\|$. The construction procedure which

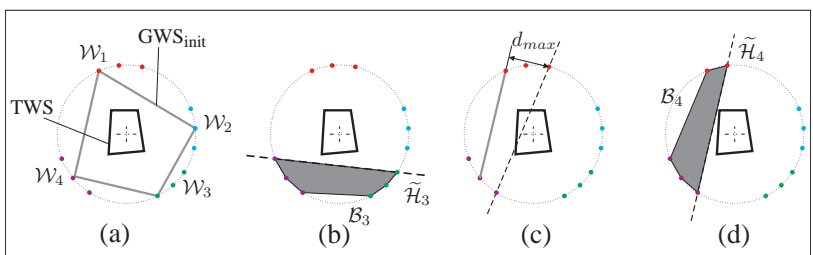

Fig. 5. Identifying restrictive wrenches: (a) Wrench sets $\mathcal{W}_{n}$, TWS and $\mathrm{GWS}_{\text {init }}$; (b) $\mathcal{H}_{3}$ linearly separates the TWS from the wrenches in $\left\{\mathcal{W}_{3}, \mathcal{W}_{4}\right\}$, (c) Wrenches in $\left\{\mathcal{W}_{1}, \mathcal{W}_{4}\right\}$ are not linearly separable from the TWS; The wrench with the largest normal distance $d_{\max }$ from the corresponding facet of $\mathrm{GWS}_{\text {init }}$ is identified; (d) Removing the restricting contact point, and thus its corresponding wrench, allows for $\widetilde{\mathcal{H}}_{4}$ to be computed successfully;

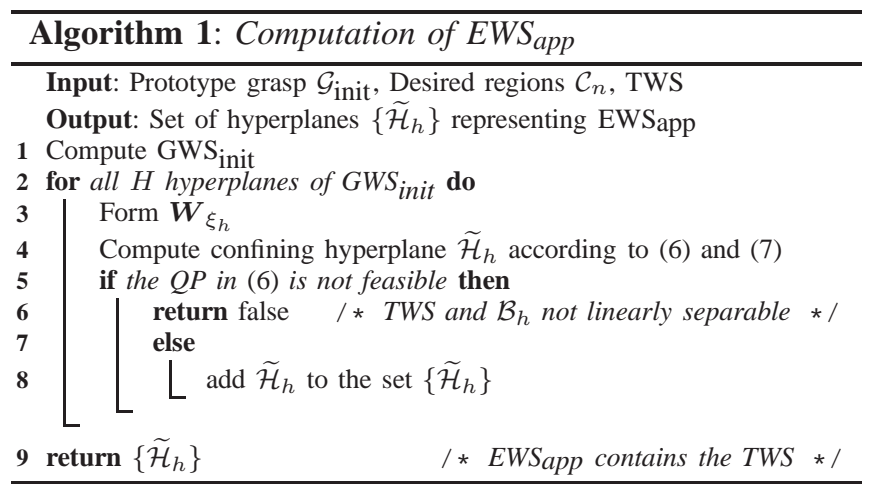

yields the EWS $\mathrm{Epp}_{\text {app }}$ in $\mathcal{H}$-representation is summarized in Algorithm 1. Invalid user-specified ICRs are detected when not all convex hulls $\mathcal{B}_{h}$ are linearly separable from the TWS, which means that some possible grasps would not be viable. A continuous spherical TWS with a specified radius can be considered by letting $\boldsymbol{W}_{\text {TWS }}$ in (6) contain the zero-wrench only. In this case, a subsequent verification if the offsets $b_{h}$ of every element in the obtained set $\left\{\widetilde{\mathcal{H}}_{h}\right\}$ are equal or larger then the given radius is necessary. Figure 10 shows an example of the sequential computation of confining hyperplanes $\widetilde{\mathcal{H}}_{h}$ for regions $\mathcal{C}_{n}$ specified on the discretized ellipse shown in Fig. 9. In this paper, the Qhull-package [15] was employed for the computation of the convex hull in the first step of the above algorithm. It has, for six-dimensional input, a complexity of $\mathcal{O}\left(N^{3} / 6\right)$ with respect to the $N$ contact points whose associated wrenches form the vertices of $\mathrm{GWS}_{\text {init }}$. An upper bound for the maximum number of facets of $\mathrm{GWS}_{\text {init }}$, and thus for the QP's to be solved in Algorithm 1, can be given as $H \leq N^{3} / 6$ [16]. Forming matrices $\boldsymbol{W}_{\xi_{h}}$ from the vertex-facet incidences of $\mathrm{GWS}_{\text {init }}$ in step 3 can be done in linear time. The results presented in Section VI were generated using an off-the-shelf QP solver [17].

\section{B. Limitations}

The above described way of finding the confining hyperplanes of the EWS $\mathrm{App}_{\mathrm{ap}}$ does not guarantee for Proposition 1 to hold true. It is possible that the number of those wrenches in $\boldsymbol{W}_{\xi_{h}}$ which act as support vectors of $\widetilde{\mathcal{H}}_{h}$ is smaller than $K$. The computation just tries to maximize the margin between the TWS and the respective convex hull $\mathcal{B}_{h}$. This can cause the EWS $\mathrm{Epp}_{\mathrm{ap}}$ to be unnecessarily limited in some areas (see Fig. 10-c). Furthermore, if not all viable grasps are similar according to Definition 1 (which cannot be verified 


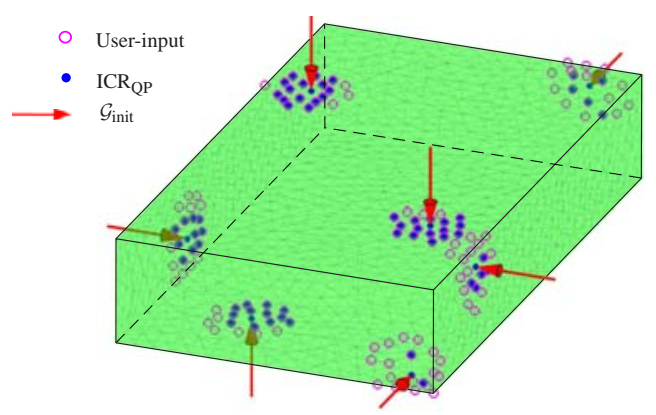

Fig. 6. Removing restrictive contact points: ICRs for a 7-fingered grasp; The regions are evaluated by removing overly restrictive contact points from the user input according to the strategy presented in Section V-A; Computation time $t_{\mathrm{QP}}=0.067 \mathrm{~s}$;

a priori), the grasp wrench spaces confining the actual EWS can comprise vertices in different wrench sets $\mathcal{W}_{n}$ than those indexed in $\xi_{h}$. Again, this can result in unnecessarily restrictive hyperplanes $\widetilde{\mathcal{H}}_{h}$.

However, the following holds true: by construction, all wrenches in $\mathcal{W}_{\xi_{h}}$ lie in the exterior half-space of the associated hyperplane $\widetilde{\mathcal{H}}_{h}$. This is equivalent to the criterion which is utilized in currently common approaches as discussed in Section II-D (points qualify for inclusion in region $\mathcal{C}_{n}$, if their corresponding wrenches lie in the intersection of exterior half-spaces associated with initial contacts $\left.\boldsymbol{p}_{g_{n}}\right)$. Thus, the method is conservative in a sense that no false positives are generated (i.e. no grasps are classified as being able to preserve a given TWS if they are not).

\section{PRIORITIZING POINTS FOR INCLUSION IN $\mathcal{C}_{n}$}

In Section IV-A, we introduced a method for approximating the space of disturbances all viable grasps, associated with given sets of contact regions $\mathcal{C}_{n}$, are able to resist. Algorithm 1 yields an approximation of the EWS which is not only conditioned on the grasp wrench space of the initial grasp, as in the state-of-the-art methods, but on the regions $\mathcal{C}_{n}$ themselves. From a user point of view, this opens up the possibility of prioritizing certain points for inclusion in the ICRs. This gives a useful technique for a number of applications, one of which is the possibility of predefining regions $\mathcal{C}_{n}$ in form of a desired distribution of contact points.

\section{A. Predefined contact regions}

Often a uniform and "circular-shaped" distribution of points within regions $\mathcal{C}_{n}$ is desired, since they can directly be related to the positioning accuracy of a robotic fixturing device. Defining such regions can be done by associating the contact points forming each region $\mathcal{C}_{n}$ with graphs, which have the corresponding initial grasp contacts $\boldsymbol{p}_{g_{n}}$ as their respective root nodes. A simple breadth-first-search (BFS) which is stopped at a predefined depth yields "layers" of contact points centered around the prototype grasp contacts. Figure 1 shows an example of such regions on the model of a parallelepiped. Algorithm 1 gives an efficient way to verify if all grasps with one contact point in each region are viable.

However, expecting the user to specify sets $\mathcal{C}_{n}$ which yield a non-empty EWS $_{\mathrm{app}}$ is a stringent requirement. In

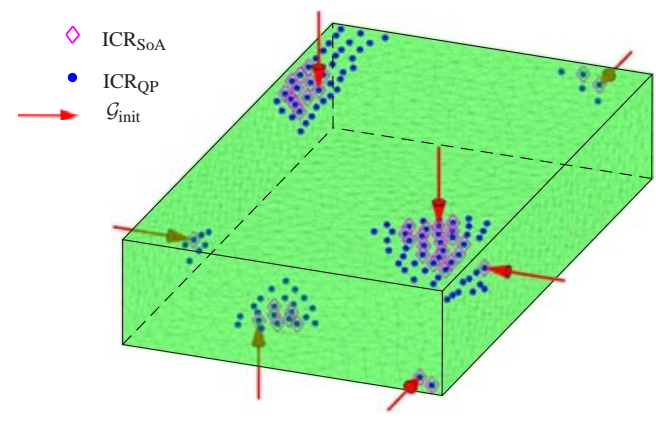

Fig. 7. Comparison of algorithms for the generation of ICRs: Regions $\mathcal{C}_{n}$ for a 7-fingered grasp; ICR $\mathrm{OP}$ denotes the regions obtained by the method detailed in Section V-B, ICR $\mathrm{SoA}_{\mathrm{A}}$ are obtained by the state-of-theart approach outlined in Section II-D;

case of Algorithm 1 failing during the computation of hyperplane $\mathcal{H}_{h}$, one possibility is to remove one (or multiple) contact point(s) from some region(s) associated with $\mathcal{W}_{\xi_{h}}$ and retry. We suggest a simple heuristics in order to decide which contact point to remove, which is depicted in Fig. 5. Removed is the contact point whose associated wrench lies in the interior half-space of the hyperplane containing the $\mathrm{h}^{\text {th }}$ facet of $\mathrm{GWS}_{\text {init }}$ and comprises the largest distance from this hyperplane. Figure 6 shows an example of regions derived via this method. Another way of ensuring feasible user input is to treat Algorithm 1 as a post-processing step of the state-of-the-art algorithm in order to improve the estimation of the EWS.

\section{B. Sequential inclusion of points in $\mathcal{C}_{n}$}

Instead of predefining the ICRs, a user can provide a logic for sequentially including points in regions $\mathcal{C}_{n}$ in order to "grow" them from the corresponding initial grasp points. Consider adding points to regions $\mathcal{C}_{n}$ according to the same breadth-first strategy as described above. However, instead of limiting the depth of the search, each time a

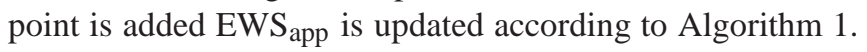

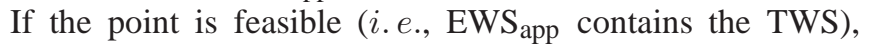
the neighbors of the point are enqueued in the search. The procedure stops when no more feasible points are found. Regions $\mathcal{C}_{n}$ can be prioritized according to their position in the sequence. In particular, choosing the sequence $n=1 \rightarrow 2,2 \rightarrow 3, \ldots, N-1 \rightarrow N$ provides an alternative way of computing ICRs which, compared to the state-ofthe-art method, yields larger regions for the same TWS as illustrated in Fig. 7.

If the position of certain initial grasping points $\boldsymbol{p}_{g_{n}}$ is known precisely, e.g. when there are several locator pins to hold a workpiece [9], it is possible to exclude the corresponding regions $\mathcal{C}_{n}$ from the sequence. This allows for more points to be included in the remaining regions. An example is shown in Fig. 8, where contact points are only added to two out of seven regions $\mathcal{C}_{n}$.

\section{Numerically Evaluated Results}

Algorithm 1 was implemented in Matlab and evaluated on a PC comprising a Core 2 Duo 2.9-GHz processor and 4GB RAM. The presented methods work for arbitrary objects, the model of a parallelepiped sampled with a number of 


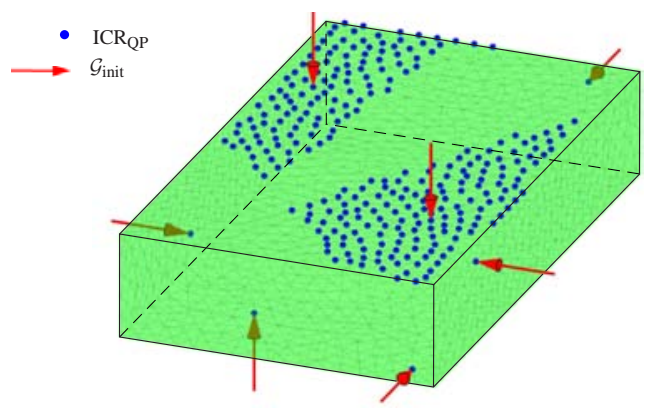

Fig. 8. Growing ICRs with some fixed grasp contacts: ICRs for a 7-fingered grasp with 5 contacts fixed beforehand;

$S=1714$ vertices, which are meshed by 3424 triangles, was used for benchmarking. For evaluation purposes hundred 7-fingered prototype form-closure grasps were randomly created for the test object. Associated to each of these grasps is a TWS represented by an origin-centered sphere with half the radius of the largest insphere of the according $\mathrm{GWS}_{\text {init }}$.

First, we evaluate the quality of the approximations

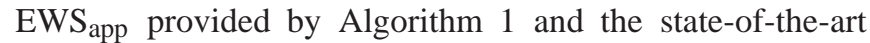
(SoA) method. For this purpose the ICR $\mathrm{IOA}_{\mathrm{So}}$, generated by the SoA approach on the test grasp set, were treated as an input for Algorithm 1. The approximations EWS $_{\text {app }}$ generated by the two methods were compared to the ground-truth EWS obtained via a brute-force computation according to (4). The results are summarized in Table I. Algorithm 1 yields vastly better results than the SoA method in terms of the average percentage difference in volume $\overline{\Delta \text { Vol }}$ from the ground truth. The polytope obtained by the SoA approach is very limited in some directions due to its strong conditioning on $\mathrm{GWS}_{\text {init }}$. Note that $b_{h_{m i n}}$, the radius of the largest origin-centered insphere of the actual EWS, was correctly identified for all test cases by the QP method.

In another experiment, ICRs were generated via the $\mathrm{QP}$ approach by sequentially adding points to regions $\mathcal{C}_{n}$ as presented in Section V-B. The flexible orientation of hyperplanes $\widetilde{\mathcal{H}}_{h}$ provided by the QP method allows for a more than three times larger average number of contact points in regions $\mathcal{C}_{n}$ compared to the SoA approach (see Table II). However, the average computation time $\overline{t_{\mathrm{QP}}}$ for the $\mathrm{QP}$ method is of two orders of magnitude higher than for the SoA method.

In a final experiment, five grasp contact points were fixed beforehand and points were sequentially added to the regions associated with the remaining two contacts as described in Section V-B. In this limited case, a point added to one region enables a number of additional grasps which equals only the cardinality of the other region considered for the inclusion of points. Thus, in each step of the sequence, the number of possible viable grasps in (3) is moderate which allows for a brute-force generation of ICRs in tolerable time. Every time a contact point is added to a region, the viability of the enabled new grasps is checked by intersecting the according grasp wrench spaces. The results of the comparison made between the brute-force computation and the QP approach are summarized in Table III. The regions generated by both methods are the same. However, regarding computational efficiency, the values for average computation time $\bar{t}$ and

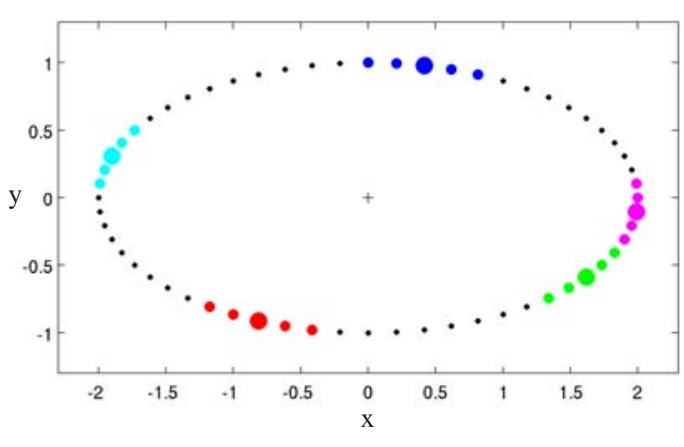

Fig. 9. Predefined ICRs on an ellipse: Uniform ICRs for a five-fingered grasp; Big dots characterize the initial prototype grasping points;

TABLE I

EWS - APPROXIMATION: COMPARISON TO THE STATE-OF-THE-ART

\begin{tabular}{r|c|c|c|c}
\hline & $\overline{\Delta \mathrm{Vol}}[\%]$ & $\sigma(\Delta \mathrm{Vol})[\%]$ & $\overline{\boldsymbol{\Delta b}}_{\boldsymbol{h}_{\min }[\%]}$ & $\sigma\left(\Delta b_{h_{\min }}\right)[\%]$ \\
\hline QP & 54.0 & 21.7 & 0.0 & 0.0 \\
\hline SoA & 99.8 & 0.4 & 43.4 & 7.5 \\
\hline
\end{tabular}

TABLE II

ICR - GENERATION: COMPARISON TO THE STATE-OF-THE-ART

\begin{tabular}{c|c|c|c|c}
\hline & $\sum \overline{\left|\mathcal{C}_{n}\right|}$ & $\sigma\left(\sum\left|\mathcal{C}_{n}\right|\right)$ & $\bar{t}[s]$ & $\sigma(t)[s]$ \\
\hline QP & 186 & 83 & 0.827 & 0.623 \\
\hline SoA & 60 & 39 & 0.008 & 0.008 \\
\hline
\end{tabular}

TABLE III

ICR - GENERATION WITH FIXED GRASP CONTACTS

\begin{tabular}{r|c|c|c|c}
\hline & $\sum \overline{\mathcal{C}_{n} \mid}$ & $\sigma\left(\sum\left|\mathcal{C}_{n}\right|\right)$ & $\bar{t}[s]$ & $\sigma(t)[s]$ \\
\hline QP & 278 & 119 & 1.926 & 7.310 \\
\hline Brute-force & 278 & 119 & 331.543 & 414.789 \\
\hline
\end{tabular}

standard deviation $\sigma(t)$ are two order of magnitudes higher for the brute force approach.

The results indicate that the algorithm introduced in this work is equivalent to a brute-force solution regarding the number of points qualified for inclusion in $\mathcal{C}_{n}$. Compared to the state-of-the-art method, it yields larger regions $\mathcal{C}_{n}$ and provides a better approximation of resistible disturbances for the price of computational efficiency.

\section{CONCLUSION}

In this work, we introduce the idea of allowing a user to influence the distribution of contact points within grasping regions on a target object's boundary. This allows incorporation of available knowledge about the targeted application. We discuss the disturbances which grasps, comprising unilateral frictionless contacts within such regions, can resist. Provided is a framework for the efficient approximation of these disturbances. Future work is directed at incorporating frictional contact constraints in order to increase the applicability of the suggested methods.

\section{ACKNOWLEDGMENTS}

This research has been partially supported by the HANDLE project, funded by the European Community's Seventh Framework Programme (FP7/2007-2013) under grant agreement ICT 231640. 


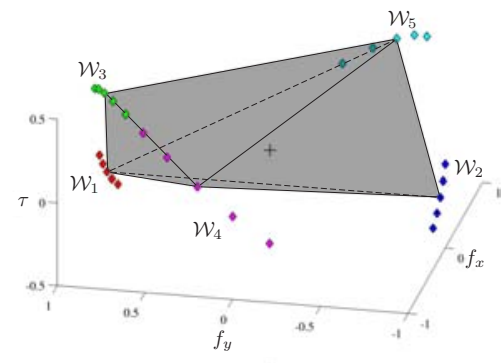

(a)

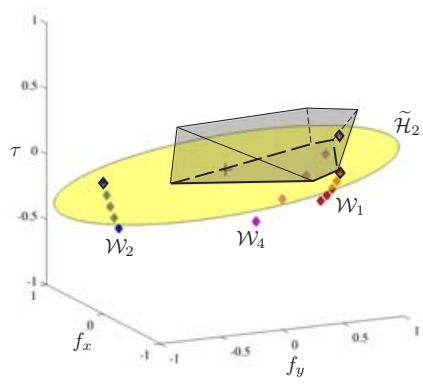

(d)

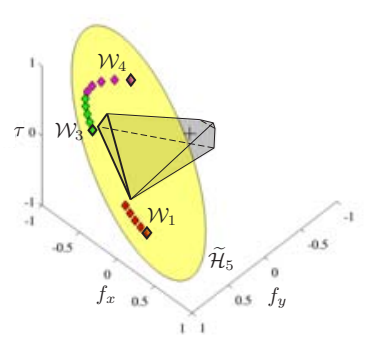

(g)

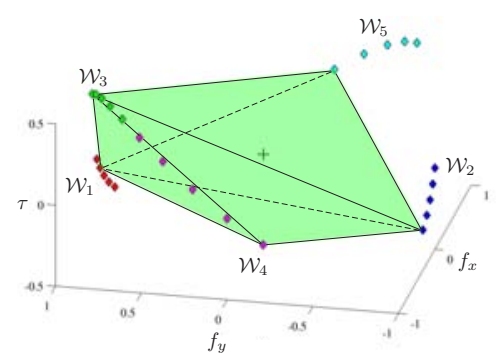

(b)

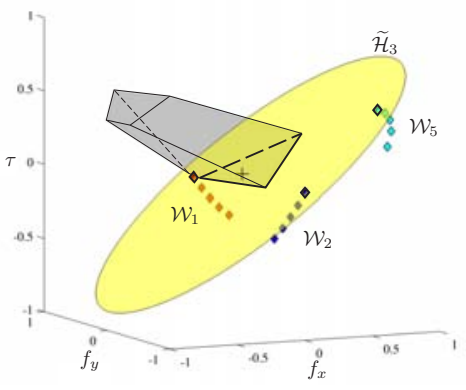

(e)

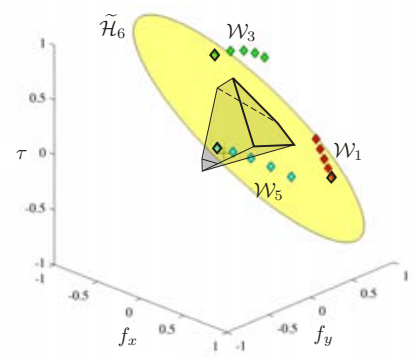

(h)

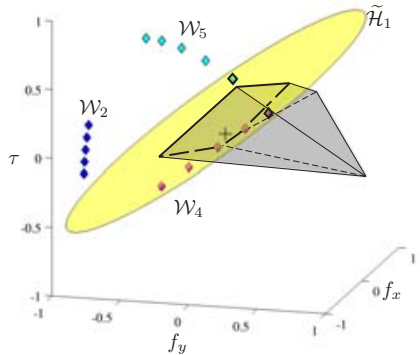

(c)

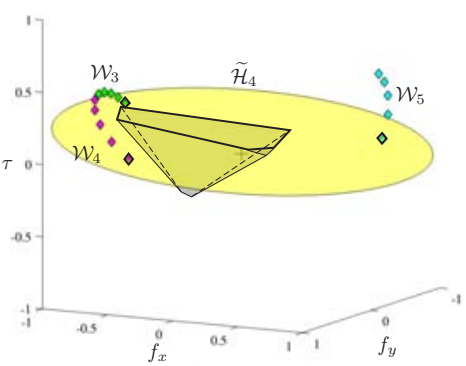

(f)

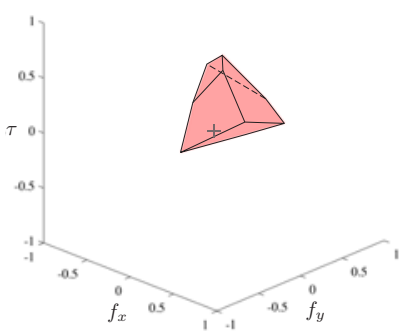

(i)

Fig. 10. Generating EWS app: Successive computation of hyperplanes $\widetilde{\mathcal{H}}_{h}$ according to Algorithm 1 for the ellipse shown in Fig. 9, the considered TWS is represented by the origin; (a) Initial grasp wrench space $\mathrm{GWS}_{\text {init }}$; (b) A viable grasp whose corresponding GWS comprises a different face lattice than $\mathrm{GWS}_{\text {init }}$ (e.g. the convex hull in (a) comprises a facet spanned by one wrench each from the sets $\mathcal{W}_{3}, \mathcal{W}_{4}$ and $\mathcal{W}_{5}$ whereas the GWS in (b) does not); Thus, the grasps corresponding to the GWS in (a) and (b) are not similar according to Definition 1; (c)-(h) Approximation of the EWS by gradually limiting GWS ${ }_{i n i t}$, the wrenches supporting the hyperplanes $\widetilde{\mathcal{H}}_{h}$ are depicted in bold frames; The hyperplane in (c) does not fulfill Proposition 1, thus $\widetilde{\mathcal{H}}_{1}$ is unnecessarily constrictive; The gray shaded polytope in (h) represents the final EWSapp; (i) The actual EWS, yielded by a brute-force computation;

\section{REFERENCES}

[1] F. Reuleaux, The Kinematics of Machinery. London: Macmillan, translated by A. B. W. Kennedy, 1876.

[2] A. Bicchi, "On the closure properties of robotic grasping," IJRR, vol. 14 , no. 4, pp. 319-334, 1995.

[3] B. Mishra, J. T. Schwartz, and M. Sharir, "On the existence and synthesis of multifinger positive grips," Algorithmica, vol. 2, pp. 541558, 1987.

[4] D. G. Kirkpatrick, B. Mishra, and C. K. Yap, "Quantitative steinitz's theorems with applications to multifingered grasping," in Proc. of the $22^{\text {nd }}$ ann. ACM Symp. on Theory of Computing, 1990, pp. 341-351.

[5] V.-D. Nguyen, "Constructing force-closure grasps," in Proc. of the IEEE Int. Conf. on Robotics and Automation, vol. 3, 1986, pp. 13681373.

[6] J. Ponce and B. Faverjon, "On computing three-finger force-closure grasps of polygonal objects," IEEE Transactions on Robotics and Automation, vol. 11, no. 6, pp. 868 -881, dec 1995.

[7] J. Ponce, S. Sullivan, A. Sudsang, J.-D. Boissonnat, and J.-P. Merlet, "On computing four-finger equilibrium and force-closure grasps of polyhedral objects," International Journal of Robotics Research, vol. 16, no. 1, pp. 11-35, 1997.

[8] N. S. Pollard, "Closure and quality equivalence for efficient synthesis of grasps from examples," IJRR, vol. 23, no. 6, pp. 595-614, 2004.

[9] M. A. Roa and R. Suárez, "Computation of independent contact regions for grasping 3-d objects," IEEE Transactions on Robotics, vol. 25, pp. 839-850, 2009.

[10] R. Krug, D. Dimitrov, K. Charusta, and B. Iliev, "On the efficient computation of independent contact regions for force closure grasps," in Proc. of the IEEE/RSJ Int. Conf. on Intelligent Robots and Systems, 2010, pp. 586-591.

[11] X. Zhu and J. Wang, "Synthesis of force-closure grasps on 3-d objects based on the q distance," IEEE Transactions on Robotics and Automation, vol. 19, no. 4, pp. 669-679, 2003.

[12] N. S. Pollard, "Parallel methods for synthesizing whole-hand grasps from generalized prototypes," Ph.D. dissertation, MIT, Dept. of Electrical Engineering and Computer Science, 1994.

[13] M. G. Ziegler, Lectures on Polytopes. Springer, 1995.

[14] B. Scholkopf and A. J. Smola, Learning with Kernels: Support Vector Machines, Regularization, Optimization, and Beyond. Cambridge, MA, USA: MIT Press, 2001.

[15] C. B. Barber, D. P. Dobkin, and H. Huhdanpaa, "The quickhull algorithm for convex hulls," ACM Transactions on Mathematical Software, vol. 22, no. 4, pp. 469-483, 1996.

[16] V. Klee, "Convex polytopes and linear programming," in Proc. of the IBM Scientific Computing Symposium: Combinatorial Problems, 1966, pp. 123-158.

[17] K. Schittkowski, "QL: A fortran code for convex quadratic programming - user's guide, version 2.11," Department of Computer Science, University of Bayreuth, Tech. Rep., 2005. 